\title{
Technique
}

\section{Scarless platysmaplasty for platysmal bands}

\author{
Melvin A. Shiffman, Sid Mirrafati \\ Consultant Plastic Surgeon, Tustin, CA, USA.
}

Address for correspondence: Melvin A. Shiffman, 1101 Bryan Ave., Suite G, Tustin, California - 92780.

E-mail: shiffmanmdjd@comcast.net

\section{ABSTRACT}

Transection of plastysmal bands has required a surgical approach that leaves scars and limits patient activities for a period of time. The author has developed a simple method to transect the platysmal bands under local anesthesia without resorting to skin incisions. The transection is performed with the use of a Vicryl@ suture that is inserted through the skin, around the platysmal band, and then out through the original entry point. A back and forth motion of the suture cuts through the band.

\section{INTRODUCTION}

latysmal banding is common in older patients (over 50), after liposuction of the neck, and in patients who have lost a fair amount of weight (over 30 pounds). Most cosmetic surgeons use a submental incision to incise the platysmal bands and at the same time suture the platysma on each side to the midline. Saylan has described a direct approach to transecting the platysmal bands through multiple small incisions in the neck. ${ }^{1,2}$ The author has been using a simple technique over the past 20 months that uses a Vicryl $^{\circledR}$ suture to transect the band under local anesthesia that can be used alone or combined with any other cosmetic neck procedure. This technique was demonstrated at a recent meeting in Germany. ${ }^{3}$

\section{TECHNIQUE}

The technique proposed utilizes a 4-0 Vicryl ${ }^{\circledR}$ suture that is used to cut the platysmal band at as many levels as necessary. The neck is marked where each of the cuts in the platysmal band is to be made, usually about $2 \mathrm{~cm}$ apart (Figure 1). The band is grasped between two fingers and held firmly making sure the whole band is included. The skin is injected with $0.5 \%$ lignocaine with epinephrine passing the needle under the band and including the skin on the opposite side of the band (Figure 2). The needle is then directed into the muscle and the muscle tumesced with the anesthetic (this is important to prevent bruising and hematoma). With the fingers still holding the band, a 4-0 Vicryl ${ }^{\circledR}$ suture is passed through the skin, under the muscle, and exits through the skin in the opposite side (Figure 3). At this point in time the band can be released from the finger pressure and the needle passed back through the exit hole (Figure 4). The needle continues subcutaneously, over the platysmal band, to exit from the original entry hole. By exerting tension on the two ends of the suture, the band is visible as trapped by the suture (Figure 5). An assistant's finger must be placed on the neck on the opposite side of the platysmal band from the suture in order to stabilize the tissue and produce some tension. The suture is 


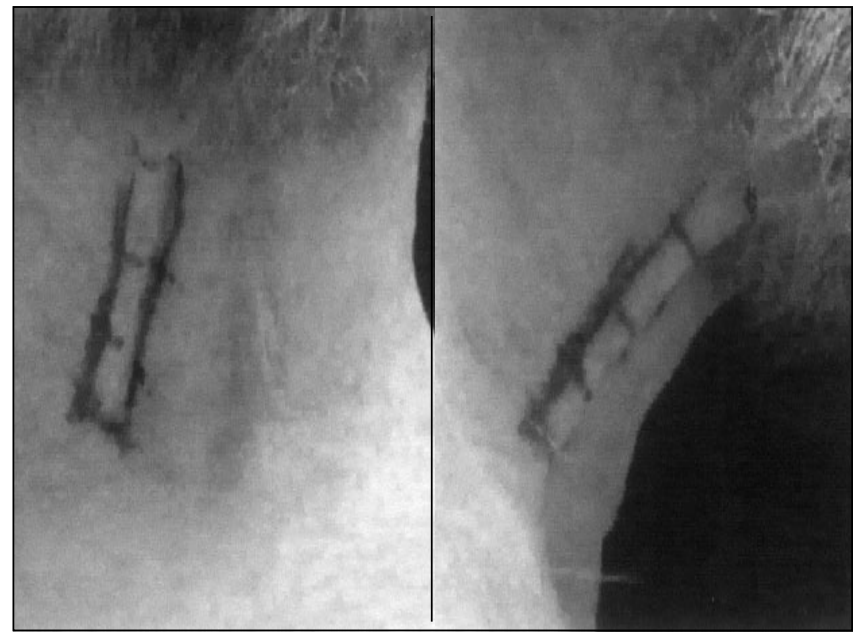

Figure 1: Limits of platysmal band is marked. Note the marks $2 \mathrm{~cm}$ apart along the band.

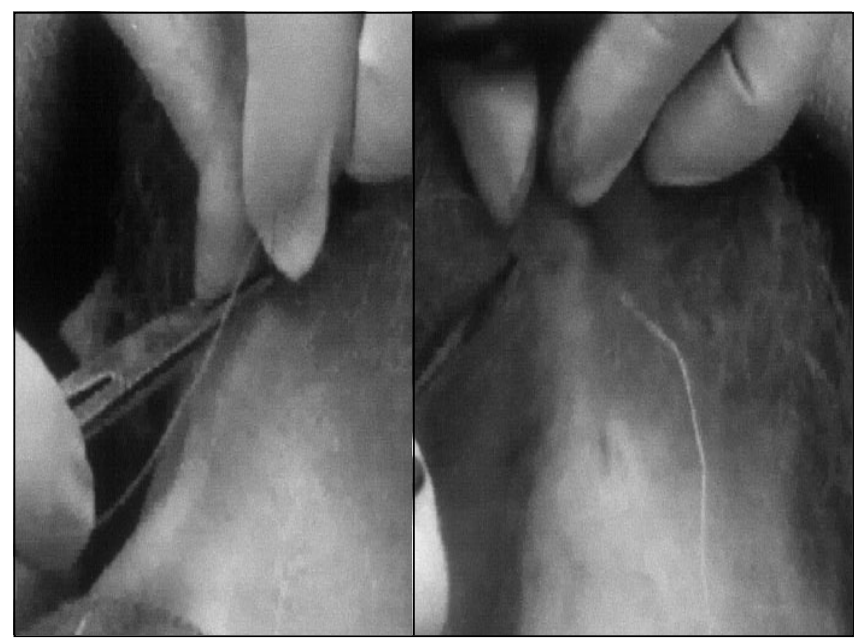

Figure 3: Inserting needle with 4-0 Vicry ${ }^{\circledR}$ through skin, under the platysmal band and through the opposite skin.

then pulled with one hand and then the other in a sawing technique, back and forth, that will cut the band and the suture will exit intact from the original entry point (Figure 6). Each of the predetermined points of transection on each side of the neck is then treated by the same technique.

Pressure with a $4 \times 4$ gauze is used over the cut platysmal band following transection for several minutes to minimize bruising (Figure 7). When all the transections have been completed, multiple folded 4 $\mathrm{x} 4$ gauze pieces are placed vertically along the platysmal band area on each side and an ace bandage or Kling dressing applied around the neck to give

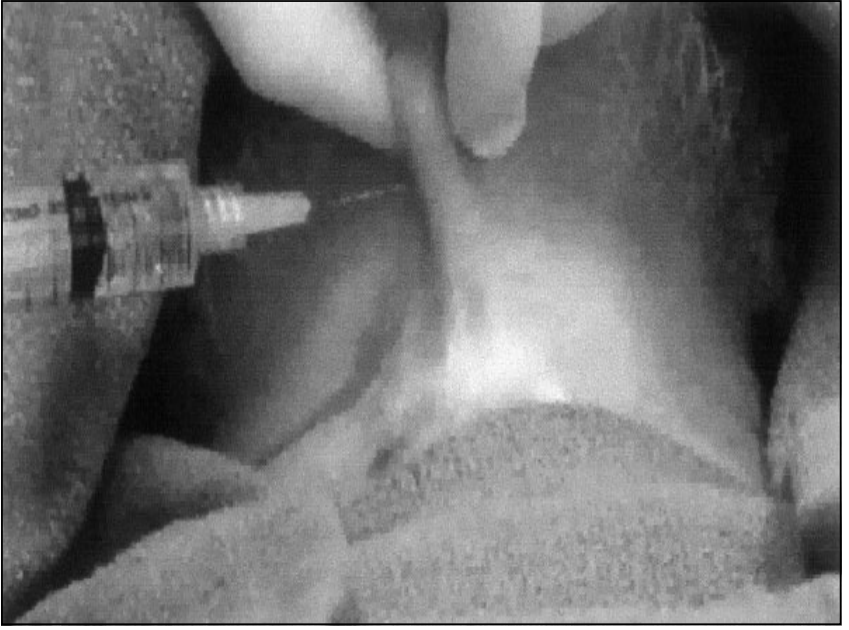

Figure 2: Injecting local anesthesia into skin and muscle

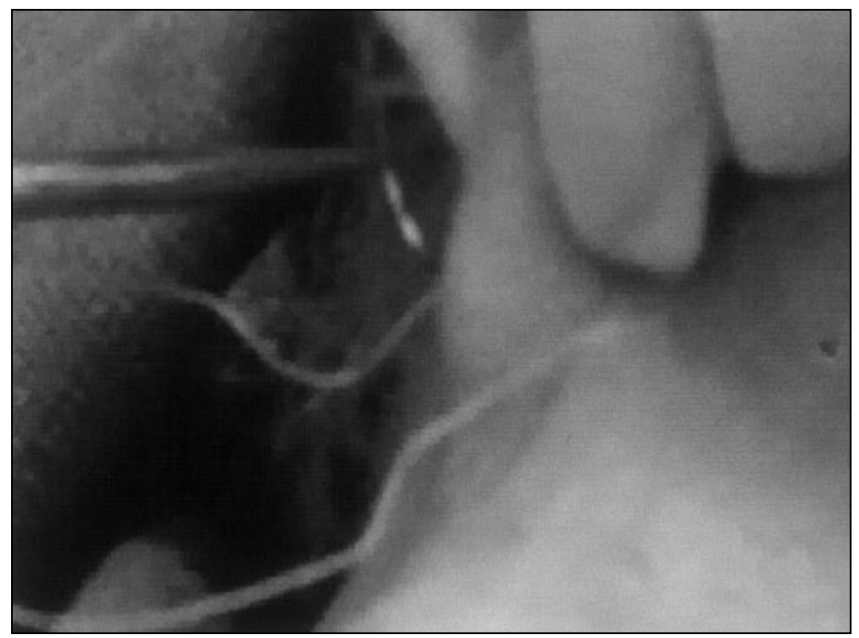

Figure 4: The needle is reversed and inserted through the exit hole.

some compression only in the region of the platysmal bands (Figure 8 ). Cold packs are used postoperatively for 24 hours. Recovery time is 24 hours after which the patient is allowed to return to normal activity. The patient is seen for follow-up on day 1 (to observe for any possible hematoma and remove all dressings), day 7 , and day 30 .

\section{DISCUSSION}

Saylan's ${ }^{1,2}$ technique involves incisions in the neck. Although the incisions are small, there is a possibility of a visible scar (Figure 9), a depression at the site of the incision is possible if very hypertrophic muscles are cut 


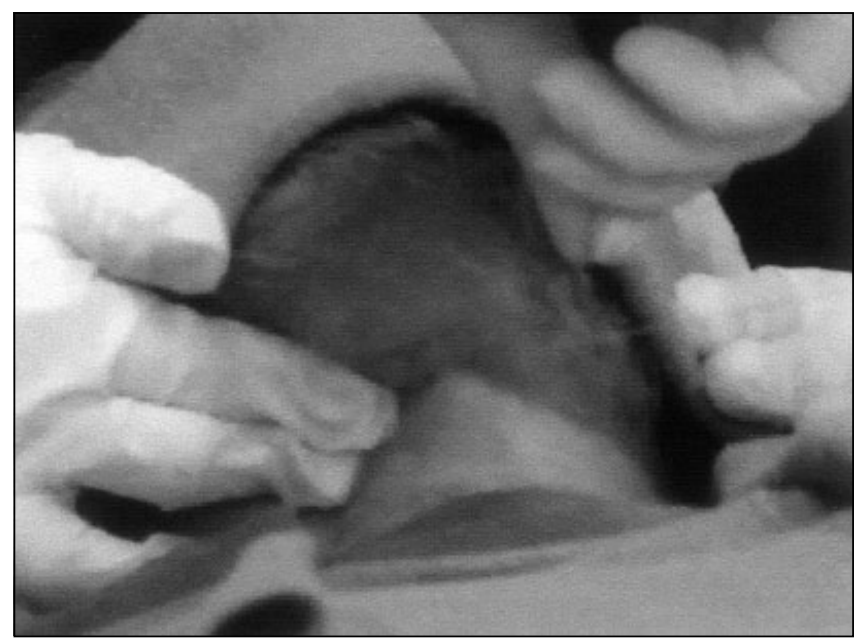

Figure 5: The suture has been brought from the exit hole subcutaneously and out the entry hole thus completely surrounding the band. Tension is placed on both ends of the suture. Note the assistant's finger holding tension on the opposite side of the platysmal band.

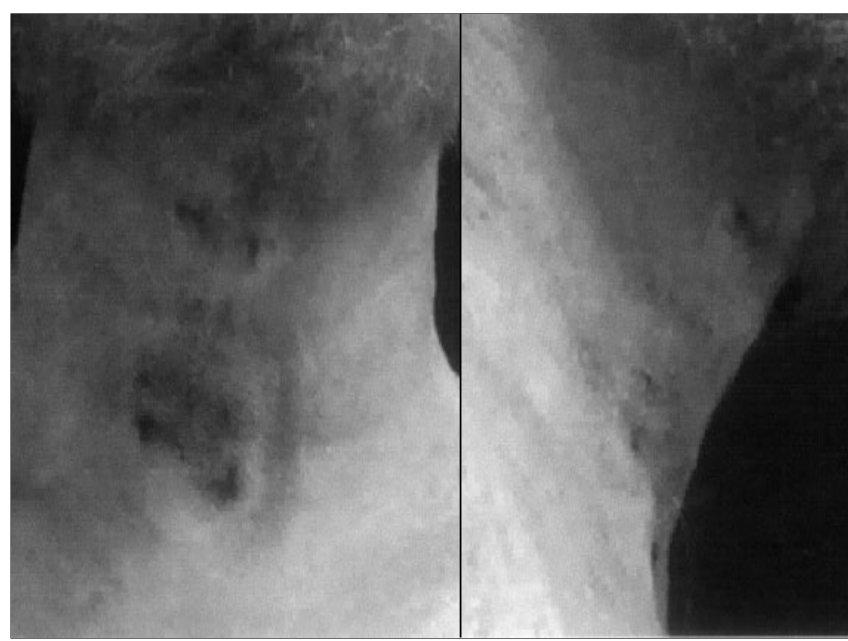

Figure 7: The neck immediately after the procedure. Note the absence of the platysmal band. Some bruising is present in this particular patient.

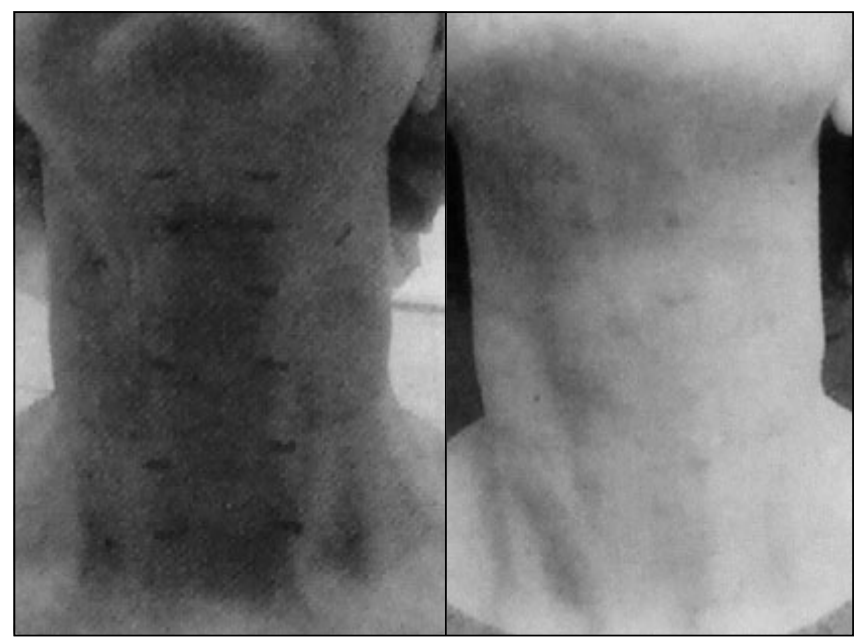

Figure 9: Scars in the neck from Saylan's technique

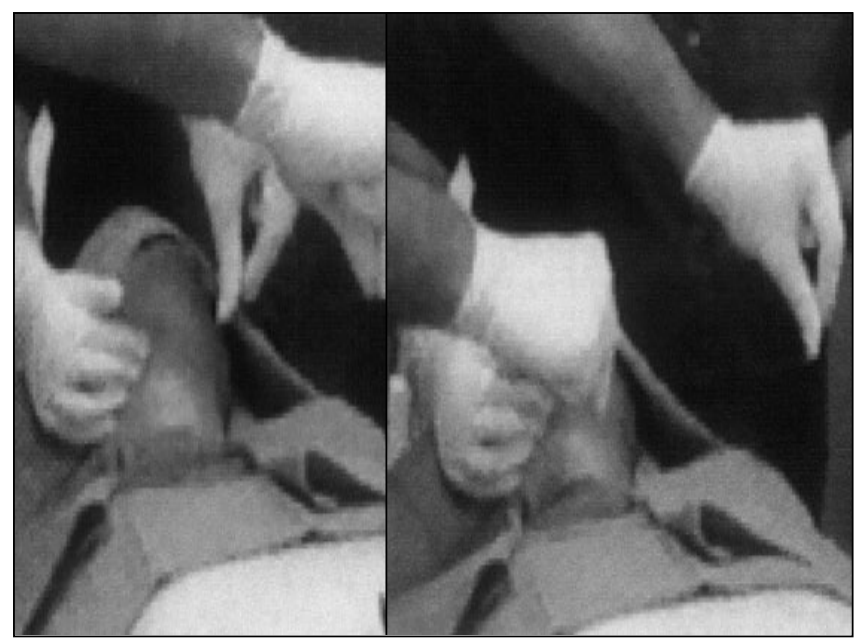

Figure 6: A sawing motion is used to transect the band and the suture is pulled through the entry hole. The suture remains intact and can be used for all the transections.

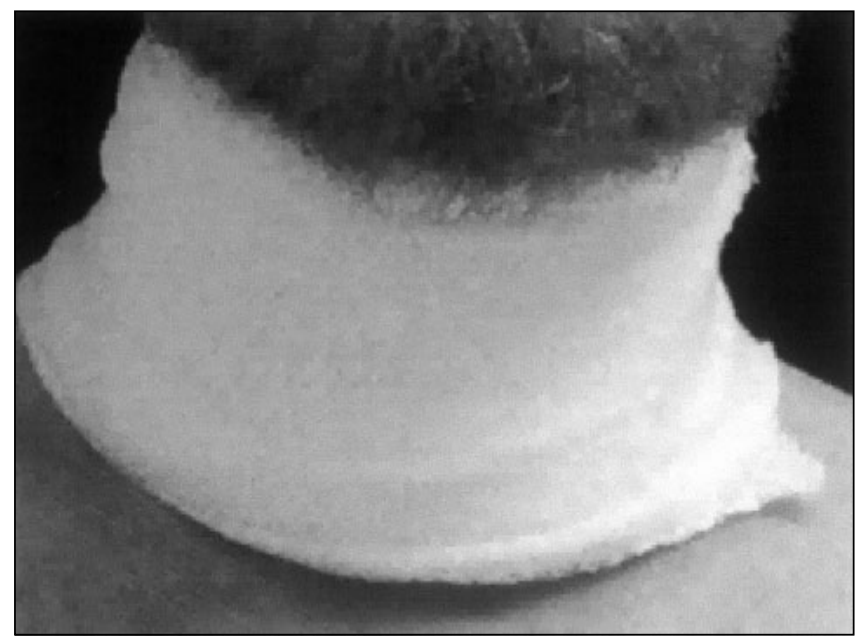

Figure 8: Compression dressing on the neck.

and more than one session may be required. Recovery time is 5-7 days. Five percent of patients had hematomas.

Sulamanidze et $\mathrm{al}^{4}$ has described a method of subcision with a wire suture that showed the wire inserted around the skin rhytid but the entry and exit holes were separated. This required care in cutting the rhytid with the wire while not cutting the skin. However, in the advertisement for the same wire K.M.I., Inc. ${ }^{5}$ (Corona, California) showed the procedure with the wire using the same entry and exit points that resolved this problem (Figure 10).

The authors have combined the Saylan and 


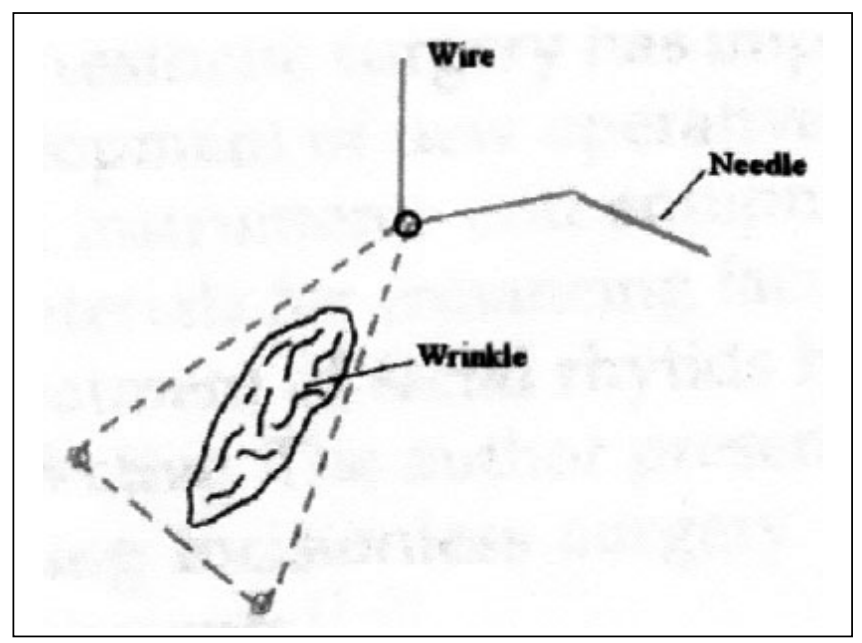

Figure 10: Sulamanidze's wire scalpel

Sulamanidze techniques to fashion the scarless Vicryl ${ }^{\circledR}$ suture platysmaplasty.

In the author's experience with 27 patients, there has been one hematoma that required aspiration for resolution (Figure 11). In this particular case, the patient had taken aspirin 36 hours prior to the procedure. Aspirin and nonsteroidal antiinflammatory drugs (NSAID) should be avoided at least 2 weeks before and two weeks after the surgery. Bruising is always a possible risk and patients should be forewarned of this problem. Make up will usually hide the bruising. There have been no infections, scars, depressions or need for repeat procedures. Patients are extremely happy with the short recovery time, minimal discomfort and no scar.

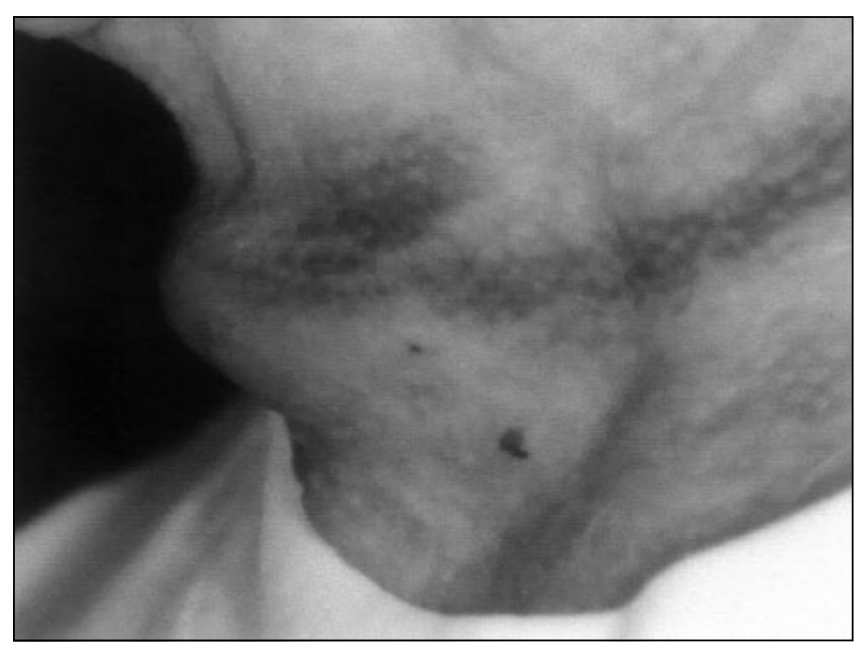

Figure 11: Postoperative hematoma that resolved with aspiration

\section{CONCLUSIONS}

The technique of suture transection of the platysmal band is unique, simple and with a short recovery time if care is taken to use local anesthetic with epinephrine to tumesce the platysmal band at the point of transection.

\section{REFERENCES}

1. Saylan, Z: Serial notching of the platysma bands. Aesthet Surg J 2001;21:412-7.

2. Saylan, Z: Serial notching of the platysma bands, update. Am J Cosm Surg 2003:20:201-4.

3. 1st Internationaler Asthetikkongress, Barth, Germany, October 2003:16-9.

4. Sulamanidze MA, Shiffman MA, Sulamanidze GM. Management of facial rhytids by subcutaneous soft tissue dissection. Int $\mathrm{J}$ Cosm Surg Aesth Derm 2000;2:255-9.

5. Subcutaneous Wire Assisted Scalpel. K.M.I., Inc. 2002. 